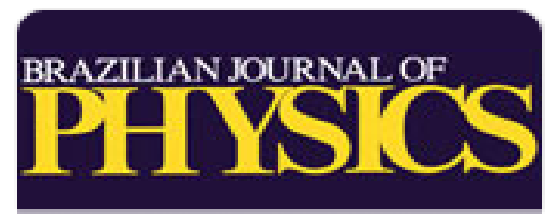

Brazilian Journal of Physics

ISSN: 0103-9733

luizno.bjp@gmail.com

Sociedade Brasileira de Física

Brasil

Akande, A.

Energetics of 2D and 3D Repulsive Hubbard Model from Their 1D Counterpart using Dimensional

Scaling for an Arbitrary Band Filling

Brazilian Journal of Physics, vol. 44, núm. 2-3, -, 2014, pp. 215-218

Sociedade Brasileira de Física

Sâo Paulo, Brasil

Available in: http://www.redalyc.org/articulo.oa?id=46431122005

How to cite

Complete issue

- More information about this article

Journal's homepage in redalyc.org

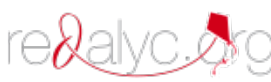

Scientific Information System

Network of Scientific Journals from Latin America, the Caribbean, Spain and Portugal

Non-profit academic project, developed under the open access initiative 


\title{
Energetics of 2D and 3D Repulsive Hubbard Model from Their 1D Counterpart using Dimensional Scaling for an Arbitrary Band Filling
}

\author{
A. Akande
}

Received: 20 August 2013 / Published online: 28 March 2014

(C) Sociedade Brasileira de Física 2013

\begin{abstract}
A dimensional scaling computation of the electron concentration-dependent ground-state energy for the repulsive Hubbard model is presented, a generalization of Capelle's analysis of the 2D and 3D Hubbard Hamiltonians with half-filled bands. The computed ground-state energies are compared with the results of mean-field and densitymatrix functional theories and of quantum Monte Carlo calculations. The comparison indicates that dimensional scaling yields moderately accurate ground-state energies close to and at half filling over the wide range of interaction strengths in the study. By contrast, the accuracy becomes poor at low filling for strong interactions.
\end{abstract}

Keywords Hubbard model · Ground-state energy · Dimensional scaling

\section{Introduction}

The Hubbard model [1-3] is a well-known prototypical Hamiltonian for a variety of strongly correlated electronic systems. The model proposes to offer qualitative insight into the mechanisms linking such distinct states as insulating, magnetic, and even novel superconducting phases to the electronic interactions in solids [4]. The Hubbard Hamiltonian $H_{\mathrm{U}}$ is defined for arbitrary dimensionality $d$. For a $d=1$ lattice of $L$ sites, for instance, in standard notation, the Hamiltonian reads

$H_{\mathrm{U}}=-t \sum_{i=1, \sigma}^{L-1}\left(c_{i+1 \sigma}^{\dagger} c_{i \sigma}+H . c.\right)+U \sum_{i=1}^{L} \hat{n}_{i \uparrow} \hat{n}_{i \downarrow}$.

A. Akande $(\square)$

School of Physics and CRANN, Trinity College, Dublin 2, Ireland e-mail: akandea@tcd.ie
The first term on the right-hand side is the kinetic Hamiltonian describes the hopping of electrons with spin $\sigma$ ( $\sigma=\uparrow, \downarrow$ ) between nearest-neighbor sites with amplitude $t>0$, while the second term, where $\hat{n}_{i \sigma} \equiv c_{i \sigma}^{\dagger} c_{i \sigma}$ is the $\sigma$-spin occupation at site $i$, accounts for the electrostatic repulsion $U>0$ in doubly occupied sites. At zero temperature, a single energy scale arises, and the ratio $U / t$ determines all electronic properties (a second energy scale would arise if an on-site energy $\sum_{i=1, \sigma}^{L} \epsilon_{i} \hat{n}_{i \sigma}$ mimicking a ionic lattice were added to $H_{\mathrm{U}}$ ). The model is parametrized by the electron concentration, or band filling, $n$, defined as the number $N$ of electrons per lattice site, i.e., $n \equiv N / L$.

Over the years, many analytical and numerical techniques were developed to deal with the model, which nonetheless still defies theorists. Exact solutions are possible in any dimensionality, via exact (Lanczos) diagonalization (ED) [5], but the scope of this approach is limited to small lattices, because the multiplicity of states in the Hilbert space associated with the $L$-site Hamiltonian is $4^{L}$. Among the non-perturbative algorithms, quantum Monte Carlo (QMC) [6] is particularly powerful. Nonetheless, QMC calculations are carried out at finite temperature, contain statistical errors, and their applicability is limited by the intrinsic fermionic sign problem. For low-dimensional systems, besides the Bethe ansatz (BA) technique [7, 8], numerical tools are available. The Density Matrix Renormalization Group (DMRG) method [9], which provides results as accurate as ED, stands out. Unfortunately, it calls for intensive computational resources. Alternative approximate methods, such as lattice density functional theory within the BA local density approximations [10, 11] have been explored in diverse successful applications [12-21].

Scaling techniques attempting to determine the properties of a system with a certain dimensionality from the known properties of the same system with smaller 
dimensionality have been proposed by Capelle and Oliveira [22], who studied the approximate dimensional scaling properties of the 1D, 2D, and 3D half-filled Hubbard model. To study the 1D system, they considered a two-site system as an artificial zero-dimensional model and obtained quantitative agreement with the exact BA solution. The total energies of the 2D and 3D models are likewise in close agreement to the QMC estimates at intermediate $U$.

An alternative test of this scaling procedure, one that has not yet been tried, is offered by the $n$ dependence of the ground-state total energy for different values of $U$. Here, we employ dimensional scaling to study on the energetics of the 2D and 3D Hubbard model at arbitrary band filling. We consider the 2D square lattice and 3D cubic lattice models with nearest-neighbor hopping. We compare the $n$-dependent energies with the results from QMC, mean-field theory, and the density-matrix theory of strongly correlated lattice fermions in Ref. [23]. Our findings establish benchmarks for the accuracy of energy computations in dimensional scaling analysis of quantum mechanical systems.

The paper is organized as follows: the next section briefly reviews the dimensional scaling technique and its approximate treatment of the Hubbard Hamiltonian. Section 3 numerically computes the ground-state energies and compares them with the results of other accurate methods in the literature. Section 4 summarizes the work.

\section{Dimensional Scaling Technique}

The virial theorem [24, 25], which relates the expectation values of the kinetic and potential Hamiltonians in eigenstates of the model Hamiltonian, can be stated in two equivalent forms: (a) the potential energy equals twice the ground-state total energy and (b) the average kinetic energy is equal to the negative of the ground-state total energy. The theorem is a remarkably useful tool that yields exact results describing otherwise unsolvable or only approximately solvable many-body problems [26]. Given two dimensionalities $d$ and $d^{\prime}$, the virial theorem immediately yields the following scaling expression relating the $d$-dimensional total energy $E_{\text {tot }}(d)$ to the associated kinetic energy $E_{\text {kin }}(d)$ and $d^{\prime}$-dimensional total energy $E_{\text {tot }}\left(d^{\prime}\right)$ and kinetic energy $E_{\text {kin }}\left(d^{\prime}\right)[22]$ :

$E_{\mathrm{tot}}(d)=E_{\mathrm{kin}}(d) \frac{E_{\mathrm{tot}}\left(d^{\prime}\right)}{E_{\mathrm{kin}}\left(d^{\prime}\right)}$.

Equation (2) shows how solutions to higherdimensional problems can be recovered from their lower-dimensional counterparts. Although the virial theorem is not applicable to the Hubbard Hamiltonian, a similar expression can be derived via the Hellmann-Feynman theorem [22]. The ground-state energy can then be written in the following form:

$E_{\mathrm{tot}}(U, d) \approx E_{\mathrm{tot}}^{D S}(U, d) \equiv E_{\mathrm{kin}}(U, d) \frac{E_{\mathrm{tot}}\left(U, d^{\prime}\right)}{E_{\mathrm{kin}}\left(U, d^{\prime}\right)}$,

The equality in (3) is only exact in the limits of small or large $U$. It is, however, approximately valid for intermediate interactions. Except for the 1D Hubbard model, closed forms for the expectation values of the kinetic energy in the ground states are not readily available. We can, however, substitute the expectation values for the kinetic energy in the noninteracting ground state for $E_{\mathrm{kin}}(U, d)$ and $E_{\mathrm{kin}}\left(U, d^{\prime}\right)$, so that (3) takes the following form:

$E_{\mathrm{tot}}(U, d) \approx E_{\mathrm{tot}}^{D S-0}(U, d) \equiv E_{\mathrm{kin}}(0, d) \frac{E_{\mathrm{tot}}\left(U, d^{\prime}\right)}{E_{\mathrm{kin}}\left(0, d^{\prime}\right)}$.

To emphasize that (4) is valid at arbitrary band filling $n$, it is convenient to substitute the following equality for (4):

$$
\begin{aligned}
E_{\mathrm{tot}}(n, U, d) & \approx E_{\mathrm{tot}}^{D S-0}(n, U, d) \\
& \equiv E_{\mathrm{kin}}(n, 0, d) \frac{E_{\mathrm{tot}}\left(n, U, d^{\prime}\right)}{E_{\mathrm{kin}}\left(n, 0, d^{\prime}\right)} .
\end{aligned}
$$

Equation (5) has been employed in Ref. [22] to estimate the ground-state energies the higher-dimensionality Hubbard models from the ground-state energies of lowerdimensionality models at half filling $(n=1)$. Since the physical properties of the Hubbard model depend on the band filling and interaction strength [7], it will be instructive to perform analogous calculations for various $n$ 's and $U / t$ 's, in order to test dimensional scaling beyond half-filled bands.

\section{Results and Discussions}

We now discuss the performance of dimensional scaling in describing the ground-state energy of the $2 \mathrm{D}$ and $3 \mathrm{D}$ Hubbard models from their 1D counterparts. We start out with $d=2$ and apply (5) to $d^{\prime}=1$ for representative $n$ 's and various $U / t$ ratios. Table 1 shows of $E_{\text {kin }}(n, 0, d)$ for four band fillings $n$ with $d=1,2$, and 3 . The resulting groundstate total energies as functions of $U / t$ are shown in the four panels of Fig. 1. For comparison, accurate QMC data, obtained at considerable computational cost [27], results from the density-matrix theory of strongly correlated lattice fermions [23] (labeled LDFT), and Hartree-Fock (HF) results are also shown.

Figure 1 shows that all methods agree at all fillings for very small $U / t$. At low band filling, the deviation separating the dimensional scaling and LDFT results increase as the electronic correlation grows. For $n=0.25$ and $n=0.5$, the ground-state total energies from dimensional scaling are only comparable with LDFT for small interactions, significant differences between the two energies arising as $U / t$ 
Table 1 Noninteracting kinetic energy $E_{\text {kin }}(n, 0, d)$ for four representative band fillings $n$ and dimensionalities $d=1,2$, and 3

\begin{tabular}{llll}
\hline$n$ & $E_{\mathrm{kin}}(n, 0, d=1)$ & $E_{\mathrm{kin}}(n, 0, d=2)$ & $E_{\mathrm{kin}}(n, 0, d=3)$ \\
\hline 0.25 & -0.49 & -0.84 & -1.01 \\
0.50 & -0.90 & -1.31 & -1.57 \\
0.75 & -1.17 & -1.56 & -1.89 \\
1.00 & -1.27 & -1.62 & -2.00 \\
\hline
\end{tabular}

increases. Closer to half filling and at half filling, i.e., for $n=0.75$ and $n=1.0$, the dimensional scaling results agree well with the LDFT curves even at high $U / t$.

We associate the substantial discrepancies on the righthand sides of the bottom panels in Fig. 1 with the metalinsulator phase transition - the Mott transition-which, for $d=2$ and 3 , occurs at intermediate $U / t \approx U_{c} / t$. For $d=$ 1 , the ground state of the Hubbard model for a half-filled band is insulating for all $U / t>0$ and conducting for $U / t=$ 0 . Away from half filling, the metallic phase arises for any interaction strength $U / t$. For large $U / t$ and small fillings, the energies of the 2D and 3D systems, which are in the insulating phases, have to be estimated from those of the 1D ground states, which are metallic. This is the source of the deviations at small fillings for large $U / t$.

Additional insight comes from Fig. 2. The solid line shows the ground-state energies resulting from dimensional scaling for $U / t=4$ as a function of the band filling $n$. The relatively small $U / t$ ratio was chosen because it allows comparison with the QMC results in Ref. [27], shown as triangles. At low filling, $n<0.45$, the solid line coincides with the dashed line representing the LDFT results,

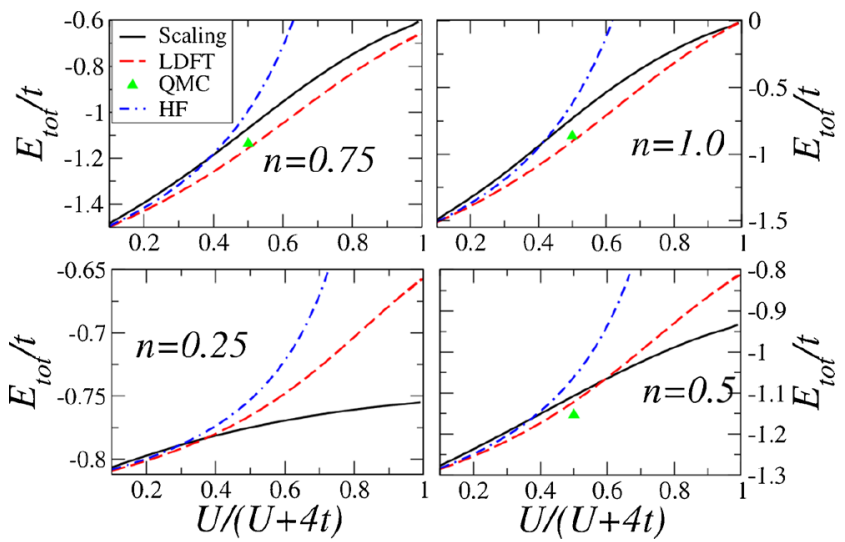

Fig. 1 (Color online) Ground-state total energy $E_{\text {tot }}$ of the Hubbard model on a 2D square lattice as a function of the Coulomb repulsion $U / t$ for various band fillings $n$. The solid curves represent our dimensional scaling calculations. The dashed lines, labeled LDFT, show the results from the density-matrix functional theory of strongly correlated lattice fermion in Ref. [23]. The triangles, labeled QMC, are the quantum Monte Carlo results in Ref. [27, 28]. The dash-dotted lines, labeled HF, represent Hartree-Fock results

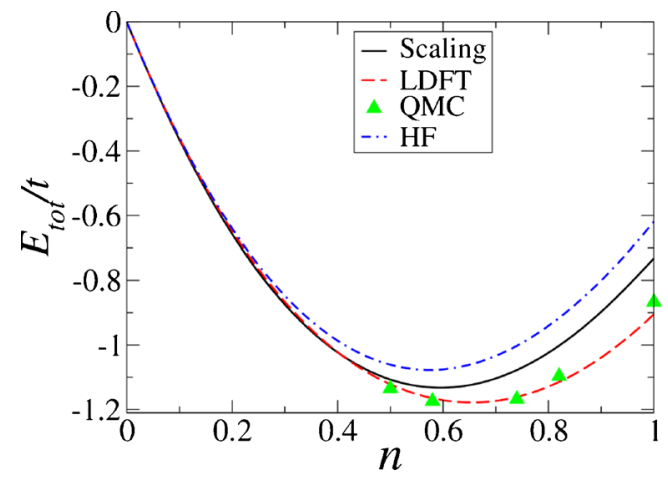

Fig. 2 (Color online) Ground-state energy $E_{\text {tot }}$ of the $U / t=4$ Hubbard model on a 2D square lattice as a function of the band filling $n$. As in Fig. 1, the solid, dashed, and dot-dashed curves and the triangles represent the results of dimensional scaling, the density-matrix functional theory in Ref. [23] (LDFT), Hartree-Fock (HF), and quantum Monte Carlo [27] computations, respectively

a congruence that is hardly surprising since we are dealing with relatively weak correlation. As the band filling grows, LDFT shows very good agreement with QMC, while the solid line displays discrepancies between dimensional scaling and QMC that are substantial and become progressively larger, even if the solid line lies closer to the more accurate calculations than the dash-dotted line representing HF.

Next, we consider $d=3$, with $d^{\prime}=1$ in (5), even though it has already been noted [22] that estimating 3D energies from the 1D ground state constitutes a drastic approximation. Figure 3 shows the ground-state energy for the 3D Hubbard model as a function of $U / t$ at four band fillings $n$ and different methods. The QMC data were computed on a $L=4^{3}$-site lattice at half filling $(n=1)$ [30]. The LDFT

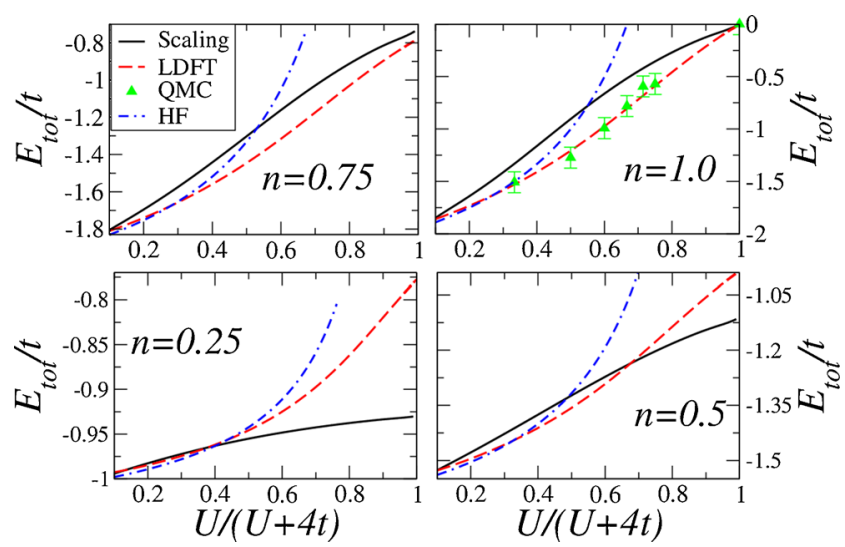

Fig. 3 (Color online) Ground-state energy $E_{\text {tot }}$ for the Hubbard model on a $3 \mathrm{D}$ cubic lattice as a function of the Coulomb repulsion $U / t$ at four band fillings $n$. The solid, dashed, and dash-dotted lines and the triangles represent dimensional scaling, the density-matrix functional theory of strongly correlated lattice fermions in Ref. [29] (LDFT), Hartree-Fock (HF), and quantum Monte Carlo (QMC) $[30,31]$ calculations, respectively 
results [29] at half filling show excellent agreement with the QMC data for all $U / t$ and therefore provide benchmarks against which the accuracy of alternative procedures can be tested at other band fillings $n$. Qualitatively, the plots reproduce the $d=2$ pattern portrayed in Fig. 1. On the one hand, the four panels indicate that, at low filling and weak interaction, the scaling approach, which is simpler and easier to implement, yields energies comparable to the results of the more accurate LDFT and QMC methods. On the other, for fillings that are close to or at half filling, the energies calculated with dimensional scaling become more accurate as $U / t$ grows.

\section{Conclusion}

The ground-state energy for the repulsive Hubbard model was calculated via dimensional scaling. The results, which sample the $U / t \times n$ parametric planes for the $2 \mathrm{D}$ and $3 \mathrm{D}$ Hubbard Hamiltonians, were obtained from the groundstate energies of the 1D model. Comparison with the accurate results of QMC and density-matrix LDFT computations shows good agreement for very weak interactions at all fillings. The largest discrepancies occur at low fillings with large $U / t$. Reasonable agreement results for the 2D and 3D Hubbard Hamiltonians at $n=1$ and $n \approx 1$ over the wide range of $U / t$ parameters in our study.

Acknowledgments The author would like to express his gratitude to Prof. Roman Lopez-Sandoval for sending his data for the densitymatrix functional theory calculations. This work is supported by the Science Foundation of Ireland under grant SFI05/RFP/PHY0062. Computational resources have been provided by the HEA IITAC project managed by the Trinity Center for High Performance Computing.

\section{References}

1. J. Hubbard, Proc. Roy. Soc. A 276, 238 (1963)

2. J. Hubbard, Proc. Roy. Soc. A 277, 237 (1964)
3. J. Kanamory, Prog. Theor. Phys. 30, 275 (1963)

4. P. Fazekas, Lecture Notes on Electron Correlation and Magnetism (World Scientific, Singapore, 1999)

5. E. Dagotto, Rev. Mod. Phys. 66, 763 (1994)

6. W. M. C. Foulkes, L. Mitáš, R. J. Needs, G. Rajagopal, Rev. Mod. Phys. 73, 33 (2001)

7. E. H. Lieb, F. Y. Wu, Phys. Rev. Lett. 20, 1445 (1968)

8. E. H. Lieb, F. Y. Wu, Physica A 321, 1 (2003)

9. U. Schollwock, Rev. Mod. Phys. 77, 259 (2005)

10. N. A. Lima, L. N. Oliveira, K. Capelle, Euro. Phys. Lett. 60, 601 (2002)

11. K. Capelle, N. A. Lima, M. F. Silva, L. N. Oliviera, in The Fundamentals of Electron Density, Density Matrix and Density Functional Theory in Atoms, Molecules and Solids, ed. by N. I. Gidopoulos, S. Wilson. Progress in Theoretical Chemistry and Physics. Kluwer Series (Kluwer, Dordrecht, 2003)

12. N. A. Lima, M. F. Silva, L. N. Oliveira, K. Capelle, Phys. Rev. Lett. 146402, 90 (2003)

13. M. F. Silva, N. A. Lima, A. L. Malvezzi, K. Capelle, Phys. Rev. B 125130, 71 (2005)

14. V. L. Jr. Campo, K. Capelle, Phys. Rev. A 061602(R), 72 (2005)

15. G. Xianlong, M. Polini, M. P. Tosi, V. L. Campo, K. Capelle, M. Rigol, Phys. Rev. B 165120, 73 (2006)

16. G. Xianlong, M. Rizzi, M. Polini, R. Fazio, M. P. Tosi, V. L. Campo, K. Capelle, Phys. Rev. Lett. 030404, 98 (2007)

17. W. Li, G. Xianlong, C. Kollath, M. Polini, Phys. Rev. B 195109, $78(2008)$

18. A. Akande, S. Sanvito, Phys. Rev. B 82, 245114 (2010)

19. A. Akande, S. Sanvito, J. Phys. Condens. Matter. 24, 055602 (2012)

20. V. V. França, D. Vieira, K. Capelle, New J. Phys. 073021, 14 (2012)

21. K. Capelle, V. L. Jr. Campo, Phys. Rep. 528, 91 (2013)

22. K. Capelle, L. N. Oliviera, Phys. Rev. B 113111, 73 (2006)

23. R. Lopez-Sandoval, G. M. Pastor, Phys. Rev. B 155118, 66 (2002)

24. E. M. Lifshitz, L. D. Landau, A Course of Theoretical Physics Vol. V: Statistical Physics (Butterworth-Heinemann, Oxford, 1980)

25. R. M. Dreizler, E. K. U. Gross, Density Functional Theory (Springer, Berlin, 1990)

26. P. Ziesche, J. Phys. C Solid State Phys. 13, 3625 (1980)

27. N. Furukawa, M. Imada, J. Phys. Soc. Jpn. 60, 3604 (1991)

28. N. Furukawa, M. Imada, J. Phys. Soc. Jpn. 61, 3331 (1992)

29. R. Lopez-Sandoval, G. M. Pastor, J. Magn. Magn. Mater. 935, 272 (2004)

30. J. Hirsch, Phys. Rev. B 35, 1851 (1987)

31. M. Ulmke, R. T. Scalettar, A. Nazarenko, E. Dagotto, Phys. Rev. B 54, 16523 (1996) 